\title{
El impacto social de la crisis de ingresos en España
}

\section{Luis Sanzo González}

Departamento de Empleo y Políticas Sociales, Gobierno Vasco

<SanzoGLu@euskadi.eus>

Artikulu honen xedea da modu labur batean aztertzea diru-sarreren krisialdiaren eragina espainiar etxebizitza mota desberdinen arabera, eta horretarako aztertzen dira 2008 eta 2014. urteen bitartean Estatistika Institutu Nazionalak biltzen dituen Bizi Baldintzen Inkestan gertatu diren aldeak. Halaber, testuak kontuan hartzen ditu diru-sarreretan izandako beherakadak ondorengo gabeziaren inguruko adierazle hauen arabera: elikadurarako arazoak, ezohizko gastuei aurre egiteko zailtasunak eta oporrez gozatu ahal izateko ezintasunak. Ondorioek nabarmentzen dute, beste datu batzuen artean, biztanleria aktiboa duen etxebizitzetan gertaturiko diru-sarreren inguruko beherakada itzela eta orokorra gertatu dela, eta kontrastea gertatzen dela aktiboa ez den biztanleria duten etxebizitzetan gertatutako joera egokiaren aldean.

\section{GAKO-HITZAK:}

Krisialdi ekonomikoa, langabezia, eraginak, dirusarreren maila, pobrezia, estatistika-datuak.
El objetivo de este artículo es analizar brevemente el impacto de la crisis de ingresos por tipo de hogar en la población española, para lo cual se examinan las variaciones observadas entre 2008 y 2014 en la Encuesta de Condiciones de Vida, que elabora el Instituto Nacional de Estadística. El texto también explora las consecuencias sociales de la caída de los ingresos en relación con tres indicadores de carencia: los problemas de alimentación, las dificultades para afrontar gastos extraordinarios y la imposibilidad de disfrutar de vacaciones. Las conclusiones ponen de manifiesto, entre otros datos, una brutal y generalizada caída de los ingresos de los hogares con población activa, en completo contraste con la tendencia favorable de los hogares de población inactiva.

\section{Palabras Clave:}

Crisis económica, desempleo, efectos, nivel de ingresos, pobreza, datos estadísticos. 


\section{Objetivo y metodología}

El objetivo de este artículo es analizar brevemente el impacto de la crisis de ingresos por tipo de hogar en la población española, para lo cual se examinan las variaciones observadas entre 2008 y 2014 en la Encuesta de Condiciones de Vida, que elabora el Instituto Nacional de Estadística. El texto también explora las consecuencias sociales de la caída de los ingresos en relación con tres indicadores de carencia: los problemas de alimentación, las dificultades para afrontar gastos extraordinarios y la imposibilidad de disfrutar de vacaciones.

La metodología aplicada en el estudio se detalla en los siguientes apartados.

\subsection{Ingresos}

Como es sabido, la información sobre ingresos contenida en la Encuesta de Condiciones de Vida se refiere a las rentas obtenidas durante el año anterior. Se comparan aquí, en este sentido, los datos de las encuestas de 2009 y 2015 (teniendo en cuenta la base actualizada hoy de aplicación). Los datos de renta utilizados en este artículo corresponden, de esta forma, a los años 2008 y 2014.

La comparación se realiza en datos corrientes. La razón es evitar las posibles distorsiones que puedan derivarse de la aplicación de una común variación asociada al IPC. En la práctica, existen distintos modelos de variación de precios en función del tipo de consumo. La aplicación del aumento del IPC medio anual observado entre 2008 y 2014 podría, por ello, introducir distorsiones importantes en este caso. Dos tipos de argumentos avalan esta aproximación. Por una parte, que una aproximación diferente tendería a dar una imagen más desfavorable de un grupo que, como la población pensionista, en especial su componente dominante de personas mayores, tiene una estructura de gasto en consumo comparativamente menos costosa que otros grupos de población. Por otra parte, que un contexto de significativo aumento o caída (aunque desigual en términos de compra/alquiler) en el precio de la vivienda, como el vivido antes y después del periodo de crecimiento, tiene un impacto diferente por grupos sociales.

La comparación se realiza, además, en términos de ingresos equivalentes per cápita, de acuerdo con la fijación de las unidades de consumo derivadas de la escala de equivalencia OCDE adaptada que utiliza Eurostat. El ingreso por unidad de consumo para cada hogar se asigna a cada miembro de éste, entendiendo que dicho ingreso es propio de cada persona del hogar.

\subsection{Grupos sociales}

La definición de los grupos sociales considerados se basa en un análisis de la variación de los ingresos entre 2008 y 2014, según relación con la actividad y deciles de renta.

Los tipos en relación con la actividad se basan en la posición de los miembros de cada hogar en relación con la actividad. En el plano personal, dicha relación se analiza vinculada a las rentas generadas, teniendo, por tanto, en cuenta la posición existente durante el año en que se generaron esas rentas, en este caso, el anterior a la realización de cada operación anual de la encuesta. Para determinar la posición dominante en términos de inactividad, ocupación y paro, se utiliza el tiempo transcurrido en cada una de esas situaciones durante el año de referencia, siguiendo este itinerario:

a. determinación de si predomina el tiempo como ocupado o no ocupado; $y$

b. si predomina la no ocupación, determinación del tiempo dominante en términos de paro o inactividad (analizando el tipo dominante 'pensionista' / 'otro tipo de inactividad económica' en este último caso).

En la determinación de hogar, se establece una tipología de mayor o menor vinculación de las personas residentes a la ocupación en el sistema productivo, de acuerdo con la siguiente clasificación:

- Hogares en los que todas las personas activas están ocupadas.

- Hogares en los que las personas activas están ocupadas o paradas.

- Hogares en los que todas las personas activas están desempleadas.

- Hogares en las que no existen personas activas. Dentro de ellos, se distinguen dos situaciones, en función de si predomina la modalidad tipo pensionista (personas jubiladas o incapacitadas) u otras formas de inactividad.

Se ha sugerido que una parte sustancial de la crisis se ha centrado en población de origen inmigrante extranjero ${ }^{1}$. Por esa razón, se ha considerado de forma separada a este colectivo, aunque únicamente en lo que respecta a aquellos hogares donde están presentes personas activas. Se distingue, en este caso, entre hogares con o sin presencia de personas ocupadas.

A la hora de identificar a la población inmigrante, el nacimiento fuera de España se utiliza como variable de referencia. Aunque esto lleva a incluir a algunas

1 De particular relevancia, en este sentido, pueden considerarse partes de la intervención del sociólogo Julio Carabaña en el XII Congreso Español de Sociología, durante el simposio 'Consecuencias sociales de la crisis y retos para la próxima década' (Gijón, 1-7-2016). En un artículo reciente (“¿Y si la desigualdad no ha crecido?", El País, 22-11-16), Carabaña volvía a señalar el papel central de la inmigración en el aumento de la pobreza severa durante la crisis: “¿Quiénes son los nuevos pobres? En términos muy aproximados, durante los primeros años de la crisis la mayor parte eran autónomos con empresas en pérdidas, que han ido dejando paso a los parados, muchos de ellos inmigrantes". 
personas de nacionalidad española por nacimiento, se trata de una distorsión menor que la asociada a la exclusión de la población de origen extranjero nacionalizada.

La variable 'tipo de hogar en relación con la actividad' (con el factor origen asociado) se analiza, a su vez, a efectos de definición de grupos sociales para el análisis, en relación con los deciles de renta por cada tipo.

Para comparar la variación de la renta media, resulta necesario, además, tener en cuenta tipos cuantitativa y sociológicamente comparables, una cuestión que se ve dificultada por el fuerte incremento del desempleo. De ahí que se haya optado por la comparación basada en la categoría de 'personas activas' / 'no activas'. El principal motivo es que la cuantía del desempleo está muy relacionada con el nivel previo de ingresos en caso de experiencia anterior de trabajo.

De acuerdo con el cruce de resultados por relación con la actividad y decil de ingresos, se delimitan los siguientes grupos de hogares, según la variación de ingresos ${ }^{2}$ :

- Hogares de personas activas con menor impacto de la caída de ingresos:

- Hogares de personas activas nacidas en España, deciles 5 a 10 (-10,3\%).

- Hogares de personas activas nacidas fuera de España, deciles 8 a 10 (-5,6\%).

- Hogares de personas activas con impacto medio o alto de la caída de ingresos:

- Hogares de personas activas nacidas en España, decil 2 (-23,4\%).

- Hogares de personas activas nacidas en España, deciles 3 a 4 (-17,3\%).

- Hogares de personas activas nacidas fuera de España, decil 3 (-23,6\%).

- Hogares de personas activas nacidas fuera de España, deciles 4 a 7 (-15,6\%).

- Hogares de personas activas con impacto muy alto de la caída de ingresos:

- Hogares de personas activas nacidas en España, decil 1 (-33,4\%).

- Hogares de personas activas nacidas fuera de España, deciles 1 a 2 (-34,5\%).

- Hogares de personas no activas con mejora del nivel de ingresos:

- Hogares de personas no activas, decil 1 (+10\%).

- Hogares de personas no activas, deciles 2 a 5 (+12,3\%).

- Hogares de personas no activas, deciles 6 a 10 (+6\%).

${ }^{2}$ La variación de la renta media entre 2008 y 2014 se señala entre paréntesis.
Además de esta clasificación, se utiliza otra más amplia para situar la participación de distintos grupos según su relación con la actividad del hogar y su pertenencia a determinados deciles de ingresos dentro de cada tipo de relación con la actividad. La clasificación ampliada es la siguiente:

- Hogares con todas las personas activas ocupadas, deciles 1 a 3.

- Hogares con todas las personas activas ocupadas, deciles 4 a 10.

- Hogares con personas activas ocupadas y paradas, deciles 1 a 2 .

- Hogares con personas activas ocupadas y paradas, deciles 3 a 6 .

- Hogares con personas activas ocupadas y paradas, deciles 7 a 10.

- Hogares con todas las personas activas paradas, deciles 1 a 3.

- Hogares con todas las personas activas paradas, deciles 4 a 5 .

- Hogares con todas las personas activas paradas, deciles 6 a 8 .

- Hogares con todas las personas activas paradas, deciles 9 a 10.

- Hogares con todas las personas no activas, deciles 1 a 2.

- Hogares con todas las personas no activas, deciles 3 a 5.

- Hogares con todas las personas no activas, deciles 6 a 10.

- Hogares con personas ocupadas, alguna nacida en el extranjero, decil 1.

- Hogares con personas ocupadas, alguna nacida en el extranjero, deciles 2 a 3.

- Hogares con personas ocupadas, alguna nacida en el extranjero, deciles 4 a 6.

- Hogares con personas ocupadas, alguna nacida en el extranjero, deciles 7 a 10.

- Hogares con personas activas paradas, alguna nacida en el extranjero, deciles 1 a 5 .

- Hogares con personas activas paradas, alguna nacida en el extranjero, deciles 6 a 7.

- Hogares con personas activas paradas, alguna nacida en el extranjero, deciles 8 a 10.

\subsection{Indicadores de carencia}

Para medir el impacto social de la crisis, se utilizan tres indicadores de carencia:

- Un primer indicador recoge el impacto relacionado con problemas de cobertura de las necesidades más básicas, reflejando problemas graves asociados al correcto acceso a la alimentación y a la vivienda, así como la cobertura de los servicios básicos (problemas de cobertura de las necesidades básicas). La existencia de estos problemas se determina cuando está presente cualquiera de las tres situaciones críticas de carencia siguientes: 
- Dos o más retrasos en el pago de la hipoteca o del alquiler del hogar en los últimos doce meses.

- Dos o más retrasos en el pago de las facturas (electricidad, agua, gas) en los últimos doce meses.

- El hogar no puede permitirse una comida de carne, pollo o pescado (o equivalentes para los vegetarianos) al menos cada dos días.

- El segundo indicador se centra en la capacidad de los hogares para acceder a un mínimo de seguridad económica para abordar gastos extraordinarios a medio o largo plazo (problemas de inseguridad económica para abordar gastos imprevistos). Estos problemas se vinculan al siguiente indicador:

- El hogar no tiene capacidad para afrontar gastos imprevistos.

- El tercer indicador se vincula a carencias que desbordan las formas graves de pobreza 0 precariedad para medir formas menores pero significativas de pérdida de bienestar (problemas de pérdida de bienestar). Se recurre, en este caso, al siguiente indicador:

- El hogar no puede permitirse ir de vacaciones fuera de casa, al menos una semana al año.

Por coherencia en las referencias temporales, los indicadores de carencia material corresponden a las Encuestas de Condiciones de Vida de 2008 y 2014 , y tienen en cuenta la estructura de renta y grupos sociales definida para el análisis. Debe recordarse que esos indicadores sí se obtienen para el año de aplicación de la encuesta.

\section{El impacto de la caída de la renta de los hogares en España}

A pesar de la recuperación económica, los ingresos medios de los hogares con presencia de personas activas se mantenían en 2014 muy por debajo de los

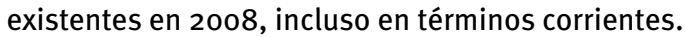
La caída es sustancial y se sitúa en el $12 \%$ (el 12,2 \% si se consideran los ingresos totales). El descenso de los ingresos medios es general en este grupo de hogares, y afecta incluso a la parte más favorecida de la sociedad. Así, la caída es del 9,8\% en el grupo que incluye a los deciles 5 a 10 de hogares con personas activas nacidas en España y afecta al $30 \%$ más acomodado de las familias de personas activas nacidas en el extranjero ( $-9,5 \%$ en ingresos totales).

Sin embargo, hay diferencias sustanciales entre los hogares con presencia de población activa. La caída es del $18,4 \%$ en las familias situadas en las decilas 2 a 4 dentro de la población nacida en España o en las decilas 3 a 7 dentro de la no nacida en España (-19\% en ingresos totales). Este descenso es aún más elevado en el grupo más desfavorecido, el de los hogares de población activa nacional situada en el decil 1 y el de las familias con personas activas nacidas fuera de España situadas en los deciles 1 y 2 . En este caso, la reducción de los ingresos medios entre 2008 y 2014 alcanza el 33,3\% (34,5\% en ingresos totales).

La caída del total de ingresos ha sido superior en las familias con población activa nacida en el extranjero $(17,3 \%)$, pero ha resultado igualmente sustancial en las familias con población activa nacida en España (11,4\%). De hecho, el 81,1\% de la caída total acumulada de ingresos corresponde a hogares de personas activas nacidas en España (Gráfico 1). En términos de ingresos medios, la disminución tampoco ha sido mayor en los hogares de población activa extranjera (un $11,7 \%$ frente a un $12,5 \%$ en los hogares de población activa nacional). Como sucede en la población general, por otra parte, al considerar los hogares según el origen de sus miembros, tanto en los hogares con presencia de personas nacidas en España como en aquellos donde no las hay se observan las mismas diferencias internas por deciles en la reducción de ingresos medios entre 2008 y 2014.

El desequilibrio en la evolución de los ingresos entre hogares de personas inactivas y activas es otra de las realidades destacables en el periodo considerado. Mientras que los ingresos medios de los hogares de personas activas caen un $12 \%$, aumentan en un $7,7 \%$ en los hogares de personas inactivas. En términos de ingresos totales agregados, la caída es del $12,2 \%$ en hogares de población activa, frente a un incremento del $11 \%$ en hogares de población inactiva. Aunque esta cuestión no se suele considerar en detalle en los estudios sobre desigualdad, supone un nuevo y evidente factor de incremento de la desigualdad interna entre la población en España.

Los aspectos señalados anteriormente se resumen en el Gráfico 2 (y, con mayor detalle, en el Anexo).

\section{Las consecuencias sociales de la caída de los ingresos de los hogares en España}

Las consecuencias sociales de la caída de ingresos observada en los hogares con personas activas en España no pueden ser minimizadas, tal y como puede observarse en los Gráficos 3 a 5, que reflejan el impacto de la crisis de ingresos en la dinámica de un grupo elegido de indicadores de carencia (método EU-SILC3/ECV). Los datos que ofrecen se centran, por una parte, en las consecuencias para los tres grandes grupos de hogares con personas activas, según su relación con la actividad, el lugar de nacimiento y el decil al que pertenecen; y por otra, en la intensidad (menor, medio-alta o muy alta) de la caída de ingresos del periodo 2008-2014. También recogen la evolución de estos indicadores de carencia en los hogares de personas inactivas.

\footnotetext{
${ }^{3}$ EU-SILC: Estadísticas Comunitarias sobre la Renta y las Condi-
} ciones de Vida (Eurostat). ECV: Encuesta de Condiciones de Vida (INE). 


\begin{tabular}{|c|c|c|c|c|c|c|c|c|c|}
\hline 5,9 & 7,6 & 15,4 & & & 2,5 & 1,3 & 8,4 & 6,7 & \\
\hline $\begin{array}{l}\text { Activas } \\
\text { ESP D } 1\end{array}$ & $\begin{array}{l}\text { Activas } \\
\text { ESP D2 }\end{array}$ & $\begin{array}{l}\text { Activas } \\
\text { ESP D3-4 }\end{array}$ & $\begin{array}{l}\text { Activas } \\
\text { ESP D5-10 }\end{array}$ & $\begin{array}{l}\text { ACTIVAS } \\
\text { nacidas en } \\
\text { España }\end{array}$ & $\begin{array}{l}\text { Activas } \\
\text { No ESP }\end{array}$ & $\begin{array}{c}\text { Activas } \\
\text { No ESP D } 3\end{array}$ & $\begin{array}{l}\text { Activas } \\
\text { No ESP }\end{array}$ & $\begin{array}{l}\text { Activas } \\
\text { No ESP }\end{array}$ & $\begin{array}{l}\text { ACTIVAS } \\
\text { no nacidas }\end{array}$ \\
\hline
\end{tabular}

D: decil. ESP: nacidas en España. No ESP: no nacidas en España.

Fuente: Elaboración propia a partir de la Encuesta de Condiciones de Vida 2009 y 2015 (Instituto Nacional de Estadística, datos de renta de 2008 y 2014).

Gráfico 2. Variación de los ingresos medios por tipo de hogar, según relación con la actividad, posición en la distribución de los ingresos y lugar de nacimiento. España, 2008-2014 (\%)

TOTAL

No activas D1

No activas D2-D5

No activas D6-D10

NO ACTIVAS

Activas No ESP D8-10

Activas No ESP D4-7

Activas No ESP D 3

Activas No ESP D1-D2

ACTIVAS NO NACIDAS EN ESPAÑA

Activas ESP D5-10

Activas ESP D3-4

Activas ESP D2

Activas ESP D 1

ACTIVAS NACIDAS EN ESPAÑA

ACTIVAS

ACTIVAS, ESP D5-10, NO ESP 8-10

ACTIVAS, ESP D2-4, NO ESP D3-7

ACTIVAS, ESP D1, NO ESP D1-2

\section{$-9,6$}

10,0

12,3

6,0

$-23,6$

$-34,5$

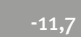

$-10,3$

$-17,3$

$-23,4$

$-33,4$

D: decil. ESP: nacidas en España. No ESP: no nacidas en España.

Fuente: Elaboración propia a partir de la Encuesta de Condiciones de Vida 2009 y 2015 (Instituto Nacional de Estadística, datos de renta de 2008 y 2014). 


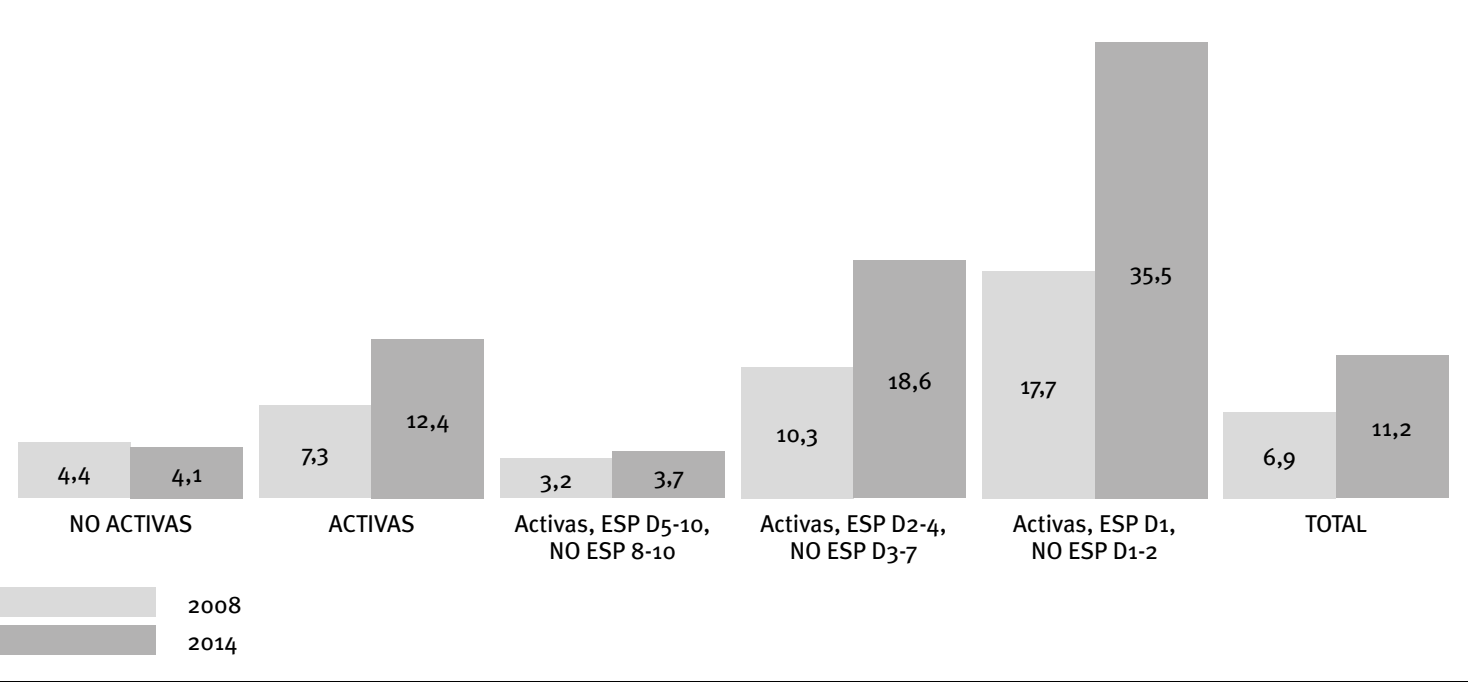

D: decil. ESP: nacidas en España. No ESP: no nacidas en España.

Fuente: Elaboración propia a partir de la Encuesta de Condiciones de Vida 2008 y 2014 (Instituto Nacional de Estadística).

Gráfico 4. Personas en hogares con problemas para afrontar gastos extraordinarios, por tipo de hogar, según relación con la actividad, lugar de nacimiento y posición en la distribución de los ingresos. España, 2008-2014 (\%)

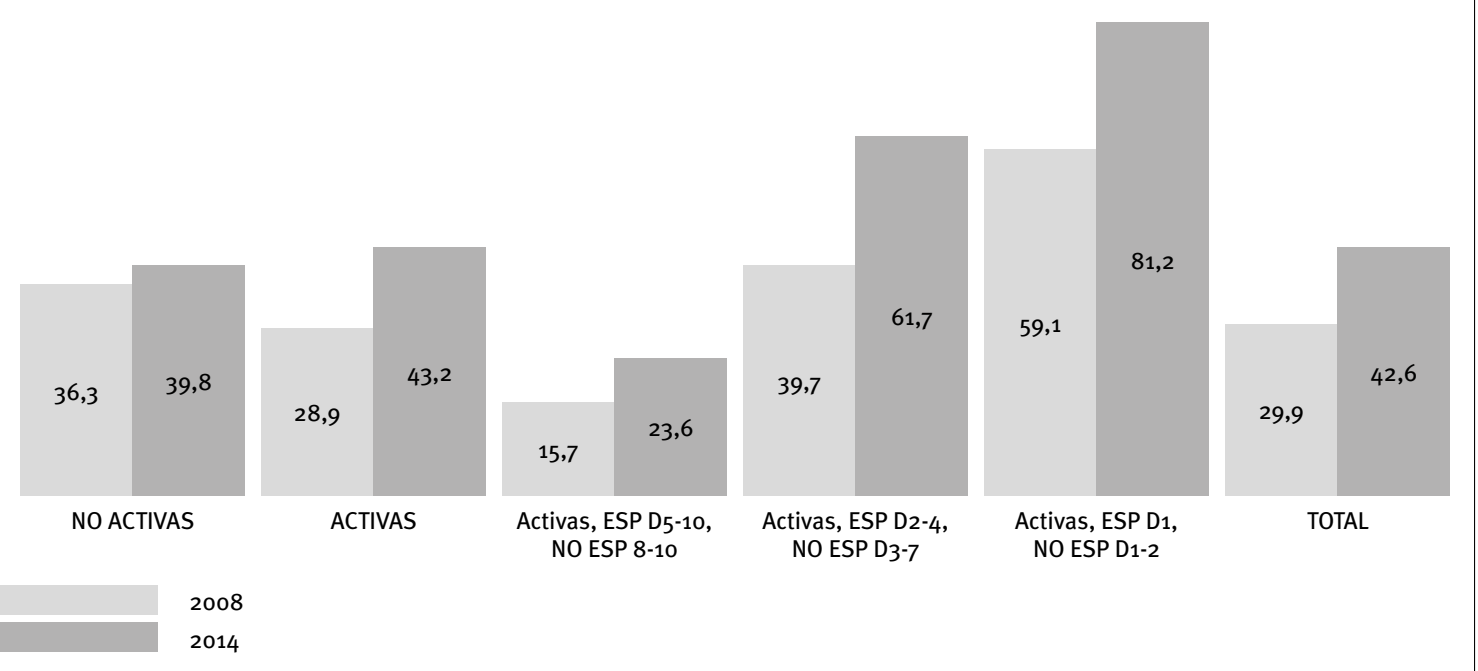

D: decil. ESP: nacidas en España. No ESP: no nacidas en España.

Fuente: Elaboración propia a partir de la Encuesta de Condiciones de Vida 2008 y 2014 (Instituto Nacional de Estadística). 


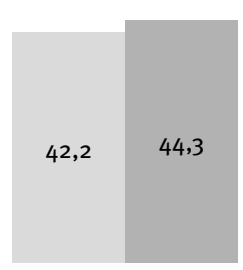

NO ACTIVAS

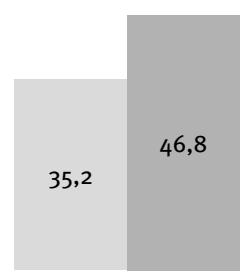

ACTIVAS

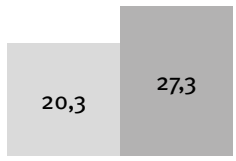

Activas, ESP D5-10, NO ESP 8-10

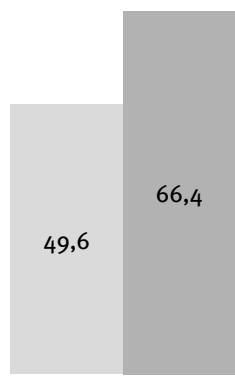

Activas, ESP D2-4, NO ESP D $3-7$

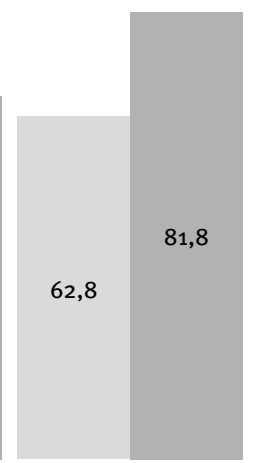

Activas, ESP D1, NO ESP D1-2

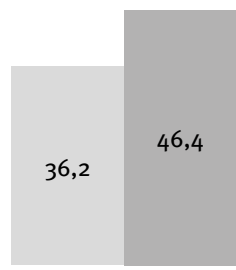

TOTAL

2008

2014

D: decil. ESP: nacidas en España. No ESP: no nacidas en España.

Fuente: Elaboración propia a partir de la Encuesta de Condiciones de Vida 2008 y 2014 (Instituto Nacional de Estadística).

\subsection{Análisis por indicadores de carencia}

\subsubsection{Problemas de alimentación}

Entre 2008 y 2004 , los problemas severos de cobertura de las necesidades básicas, los más directamente ligados a las formas graves de pobreza, aumentan de forma muy significativa: pasan de afectar a 3,12 millones de personas en 2008 , un $6,9 \%$ del total, a 5,13 millones en 2014 , un $11,2 \%$ del total, con un incremento de 4,3 puntos porcentuales. En este caso, la incidencia del problema es claramente selectiva:

- Entre los hogares de personas inactivas, se reduce del $4,4 \%$ al $4,1 \%$, en contraste con el aumento del $7,3 \%$ al $12,4 \%$ entre las personas pertenecientes a hogares de personas activas.

- El incremento es limitado entre los hogares de población activa que menos sufren la caída de los ingresos. En este grupo más favorecido de hogares activos, la prevalencia de los problemas graves de cobertura de las necesidades básicas sólo aumenta de un 3,2 \% en 2008 a un 3,7\% en 2014 .

- En cambio, el corte es brutal en los hogares de población activa que más sufren la crisis de ingresos, donde la prevalencia de las situaciones graves de cobertura de las necesidades básicas prácticamente se dobla. En el grupo con una caída de ingresos de tipo medio o alto, la incidencia de los problemas señalados pasa de $10,3 \%$ a $18,6 \%$; en el grupo que sufre una caída muy alta, la incidencia pasa del $17,7 \%$ al 35,5\%.

- En el conjunto de los hogares de personas activas, el deterioro del periodo 2008-2014 se traduce en un aumento de 1,99 millones de personas afectadas por los problemas graves de cobertura de las necesidades básicas en España.

\subsubsection{Dificultades para afrontar gastos extraordinarios}

Como es sabido, la principal consecuencia de la crisis social del periodo 2008-2014 en España es una radical caída de la seguridad económica de unos hogares que recurren con frecuencia a ahorros previos para hacer frente a la pérdida de ingresos, tratando así de mantener el consumo habitual en la medida de lo posible. La incidencia de las dificultades para abordar gastos imprevistos aumenta de un $29,9 \%$ en 2008 a un $42,6 \%$ en 2014 , con un incremento de 12,7 puntos porcentuales.

La prevalencia del problema es generalizada, aunque continúa difiriendo claramente según los grupos. En el caso de los hogares de personas inactivas, la incidencia sube del $36,3 \%$ al $39,8 \%$ entre 2008 y 2014. Sin embargo, partiendo de niveles más bajos en 2008 (28,9\%), la proporción de personas afectadas en hogares de personas activas llega al $43,2 \%$ en 2014 , con un aumento de 14,3 puntos.

El incremento es de 7,9 puntos (de 15,7\% a 23,6\%) en el grupo de hogares de población activa que menos sufren la caída de los ingresos, incremento que contribuye a una tercera parte de los alrededor de 22 puntos porcentuales que aumenta el indicador en los grupos de activos más afectados. En los hogares con una caída de ingresos de tipo medio o alto, la incidencia de estos problemas pasa de ser minoritaria en 2008 (39,7\%) a afectar a un 61,7\% de la población del grupo. En los que sufren una caída 
muy alta de sus ingresos, la incidencia pasa del $59,1 \%$ al $81,2 \%$.

En el conjunto de los hogares de personas activas, el deterioro del periodo 2008-2014 se traduce en un aumento de 5,53 millones de personas afectadas por los problemas relacionados con la insuficiencia de ingresos para poder afrontar posibles gastos extraordinarios.

\subsubsection{Imposibilidad de disfrutar de una semana de vacaciones al año}

Entre 2008 y 2014 , la proporción de personas en hogares que no pueden disfrutar de una semana de vacaciones al año aumenta en España del 36,2 \% al 46,4\%, con una dinámica evolutiva claramente correlacionada con los cambios en los indicadores de seguridad económica. De nuevo, el impacto del problema durante la crisis es netamente superior entre las personas que viven en hogares de población activa. En el caso de los hogares de personas inactivas, el indicador aumenta de 42,2 \% a 44,3\% de la población del grupo; en el de los hogares de población activa, lo hace del 35,2 \% al 46,8\%, superando el nivel correspondiente a la población no activa.

Dentro de los hogares de personas activas, las tendencias son igualmente similares a las que se observan en el indicador de seguridad económica. El deterioro es de 7 puntos entre los hogares que menos sufren la caída de los ingresos (de 20,3\% a $27,3 \%$ ), frente a incrementos de entre los 17 y 19 puntos porcentuales en los colectivos más precarios. En los hogares con una caída de ingresos de tipo medio o alto, la incidencia de este problema pasa del $49,6 \%$ al $66,4 \%$; en los que sufren una caída muy alta, la incidencia pasa del $62,8 \%$ al $81,8 \%$.

\subsection{Análisis detallado}

Es importante señalar, en este apartado, quelas consecuencias sociales de la crisis no se han concentrado en exclusiva en la población nacida fuera de España. En los hogares de personas activas, se vincula directamente a hogares de personas nacidas en España un 61,9\% del incremento observado en el volumen de población afectada por problemas graves de cobertura de las necesidades básicas (Gráfico 6). La proporción es del 82,4\% si se considera el incremento de población con problemas de inseguridad económica para hacer frente a gastos imprevistos.

La recuperación económica reciente ha permitido reducir la prevalencia de los problemas graves de cobertura de las necesidades básicas del 11,2 \% de 2014 al 10,4\% de 2015. Pero la cifra aún está muy lejos del registro de 2008 , un 6,9\%, que entonces ya resultaba bastante elevado. A pesar del periodo de crecimiento, y en parte por las características que adoptó, con una
Gráfico 6. Proporción que corresponde a la población nacida en España en el incremento de personas con carencias en hogares de personas activas, por tipo de carencia. España, 2008-2014 (\%)
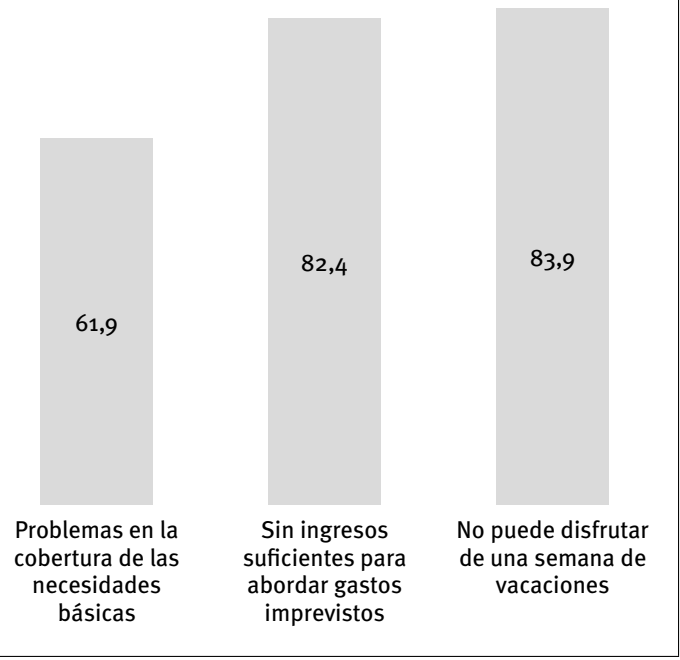

Fuente: Elaboración propia a partir de la Encuesta de Condiciones de Vida 2008 y 2014 (Instituto Nacional de Estadística).

inmigración masiva, las formas más graves de pobreza afectaban entonces a millones de personas, 3,12 en concreto. La cifra actual es de 4,8 millones de personas, un $53,7 \%$ más. Esta cifra basta para poner en evidencia el tremendo impacto social de la crisis.

Este impacto, además, no se distribuye de forma equilibrada entre los distintos grupos de población. Si la proporción es del 4,2 \% entre la población residente en hogares de personas inactivas, es del $8,9 \%$ en personas en hogares de población activa nacida en España y del $24,3 \%$ en hogares con población activa no nacida en España. A pesar de impacto diferencial en la población extranjera, un $65,8 \%$ de los casos graves detectados en 2015 corresponden a hogares de población nacida en España. Es imposible, por tanto, vincular la crisis sólo a la población extranjera.

El análisis detallado de los grupos más afectados revela la naturaleza real de los problemas graves relacionados con la cobertura de las necesidades básicas que existen en la España actual. Los principales elementos reseñables en 2015 son los siguientes (Gráfico 7):

- Más de un $40 \%$ de la población sufre problemas en la cobertura de las necesidades básicas en el conjunto de hogares con todos sus miembros activos parados y al menos alguno de ellos nacido fuera de España. En esta situación se encuentran igualmente, sin embargo, las personas vinculadas a los deciles 1 a 3 en hogares de personas activas desempleadas y nacidas en España.

- En los deciles 4 y 5 de hogares con todos sus miembros activos desempleados y nacidos en 
España, la presencia de estos problemas llega todavía al $26,9 \%$. Se encuentran igualmente afectados entre el $26,3 \%$ y el $28,3 \%$ de algunos deciles de hogares con población activa parada pero con presencia de al menos alguna persona ocupada. En concreto, se trata de los deciles 1 a 6 de hogares de este tipo con alguna persona nacida en el extranjero, y de los deciles 1 y 2 de hogares de este tipo con personas activas nacidas en España.

- En conjunto, entre la población en hogares de personas activas donde todas ellas son desempleadas y hay personas nacidas en el extranjero, o que pertenecen a los deciles 1 a 5 de hogares con personas nacidas en España, un $40,6 \%$ de las personas tienen problemas graves relacionados con la cobertura de las necesidades básicas. Por lo que respecta a los hogares con presencia de personas ocupadas y paradas, el grupo equivalente, reducido a los deciles 1 y 2 en el caso de población en hogares de personas nacidas en España, estos problemas sociales graves afectan al $27,1 \%$ de la población.

- En otros hogares de población activa nacida en España, la prevalencia de dificultades de cobertura de las necesidades básicas es aún muy elevada, de en torno al 14,5\%. Así ocurre en los deciles 3 a 6 de hogares de población con personas tanto ocupadas como paradas, así como en los deciles 6 a 8 de personas activas todas ellas desempleadas.

- Entre los hogares de personas inactivas, los problemas más graves de cobertura de las necesidades básicas no han desaparecido por completo, con un $18,5 \%$ de personas afectadas en el decil 1 de este tipo de hogares.

- En presencia de todas las personas activas ocupadas, la incidencia del indicador cae siempre por debajo del $10 \%$, pero es aún del $8,9 \%$ en los deciles 1 a 3.

Gráfico 7. Personas en hogares sin acceso a una semana de vacaciones al año, por tipo de hogar, según relación con la actividad, lugar de nacimiento y posición en la distribución de los ingresos. España, 2008-2014 (\%)

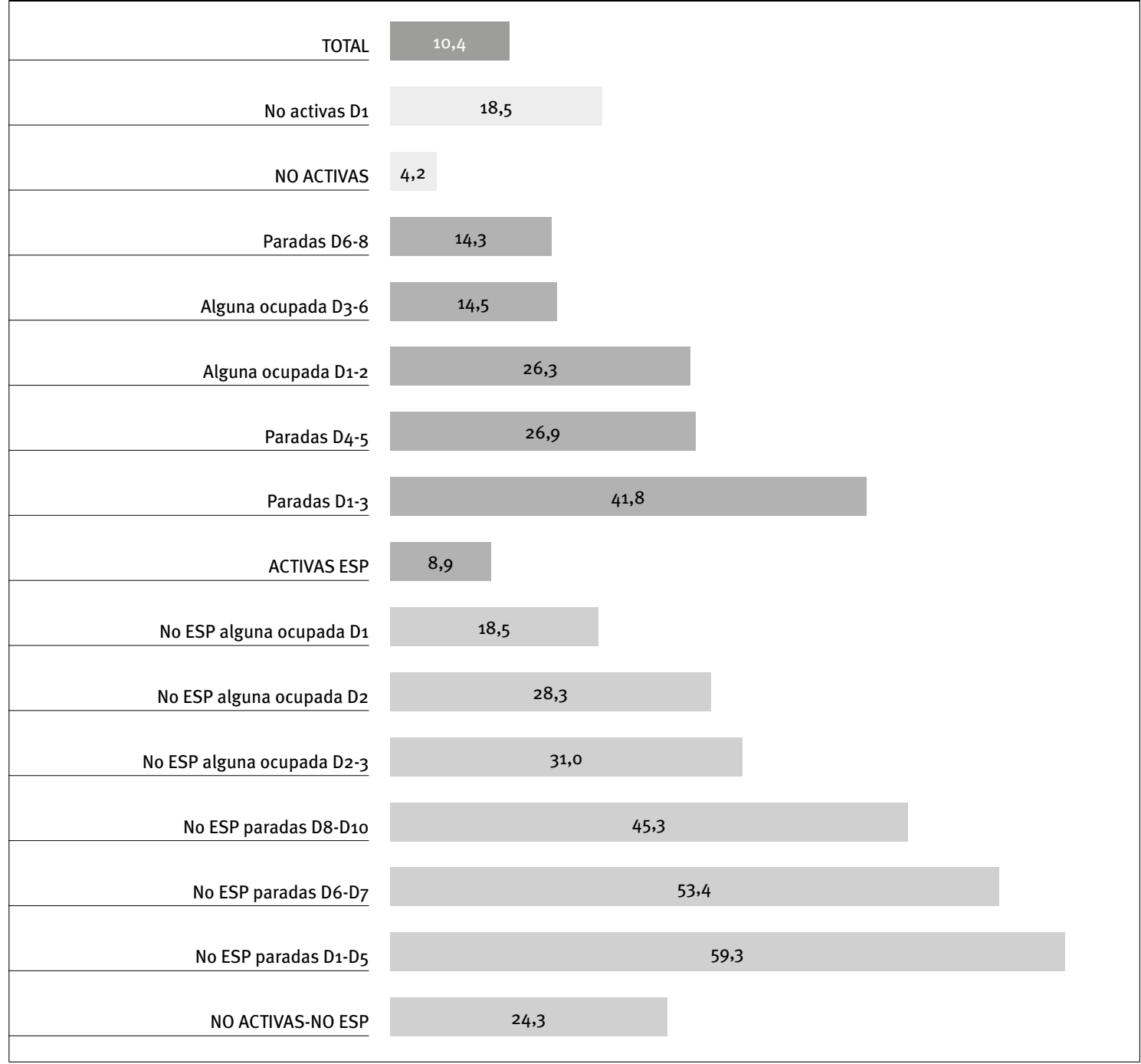

D: decil. ESP: nacidas en España. No ESP: no nacidas en España.

Fuente: Elaboración propia a partir de la Encuesta de Condiciones de Vida 2015 (Instituto Nacional de Estadística). 


\section{El nuevo perfil del grupo más desfavorecido}

En una aproximación global al conjunto de los hogares en España, se constata que la evolución de los ingresos ha provocado un significativo cambio en la estructura interna de los grupos más desfavorecidos, los que componen los deciles 1 a 3, tal y como puede comprobarse en los Gráficos 8 y 9.
Las principales líneas de cambio son las siguientes:

- En primer lugar, la proporción de personas en hogares cuyos miembros son inactivos disminuye de forma destacada en los grupos más desfavorecidos. Entre 2008 y 2014, su peso pasa del $18,5 \%$ al $6,1 \%$ en el decil 1 , y del $22 \%$ al $15,7 \%$ en los deciles 1 a 3 .

- En cuanto a los hogares con personas activas, predominan aquellos en los que está presente

Gráfico 8. Cambios en la distribución del decil 1, por tipo de hogar, según relación con la actividad, posición en la distribución de los ingresos y lugar de nacimiento. España, 2008-2014 (\%)

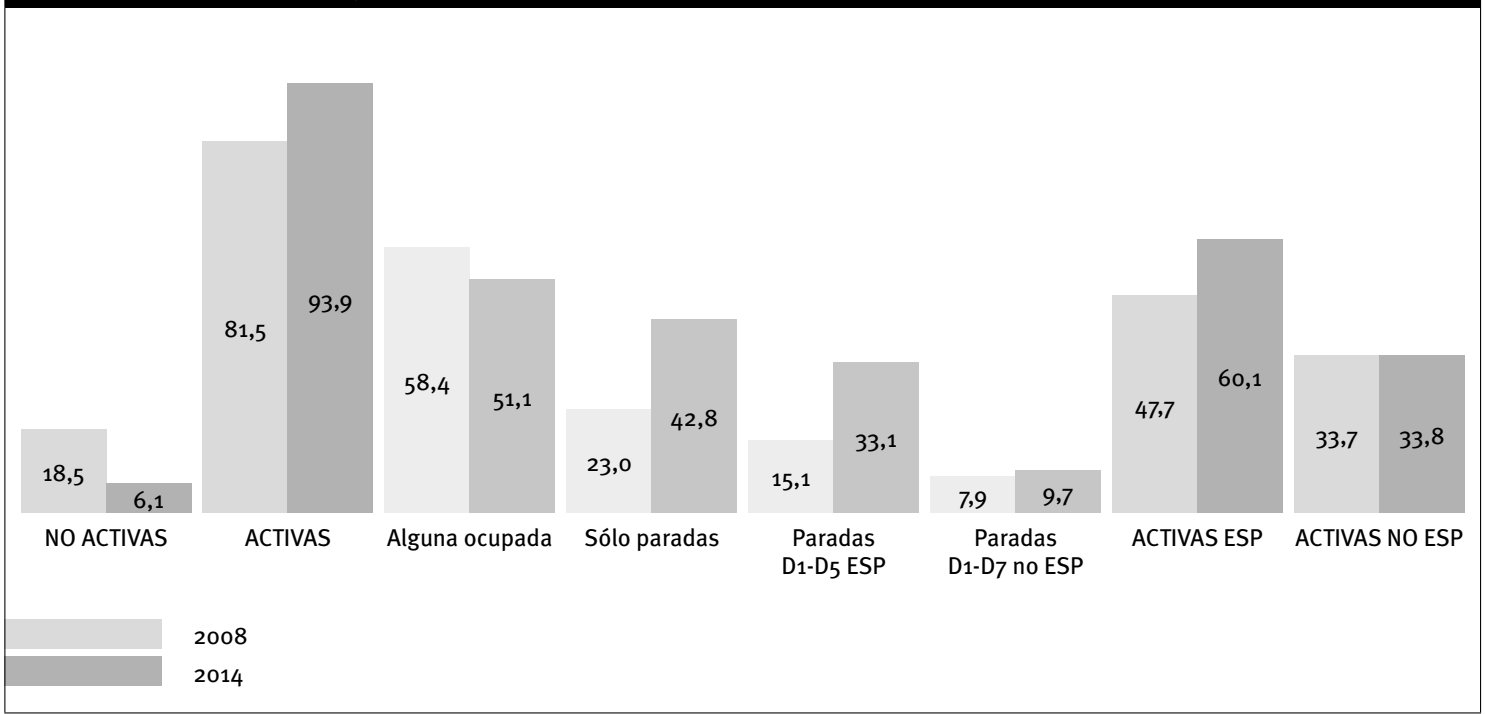

D: decil. ESP: nacidas en España. No ESP: no nacidas en España.

Fuente: Elaboración propia a partir de la Encuesta de Condiciones de Vida 2009 y 2015 (Instituto Nacional de Estadística, datos de renta de 2008 y 2014).

Gráfico 9. Cambios en la distribución de los deciles 1 a 3, por tipo de hogar, según relación con la actividad, posición en la distribución de los ingresos y lugar de nacimiento. España, 2008-2014 (\%)

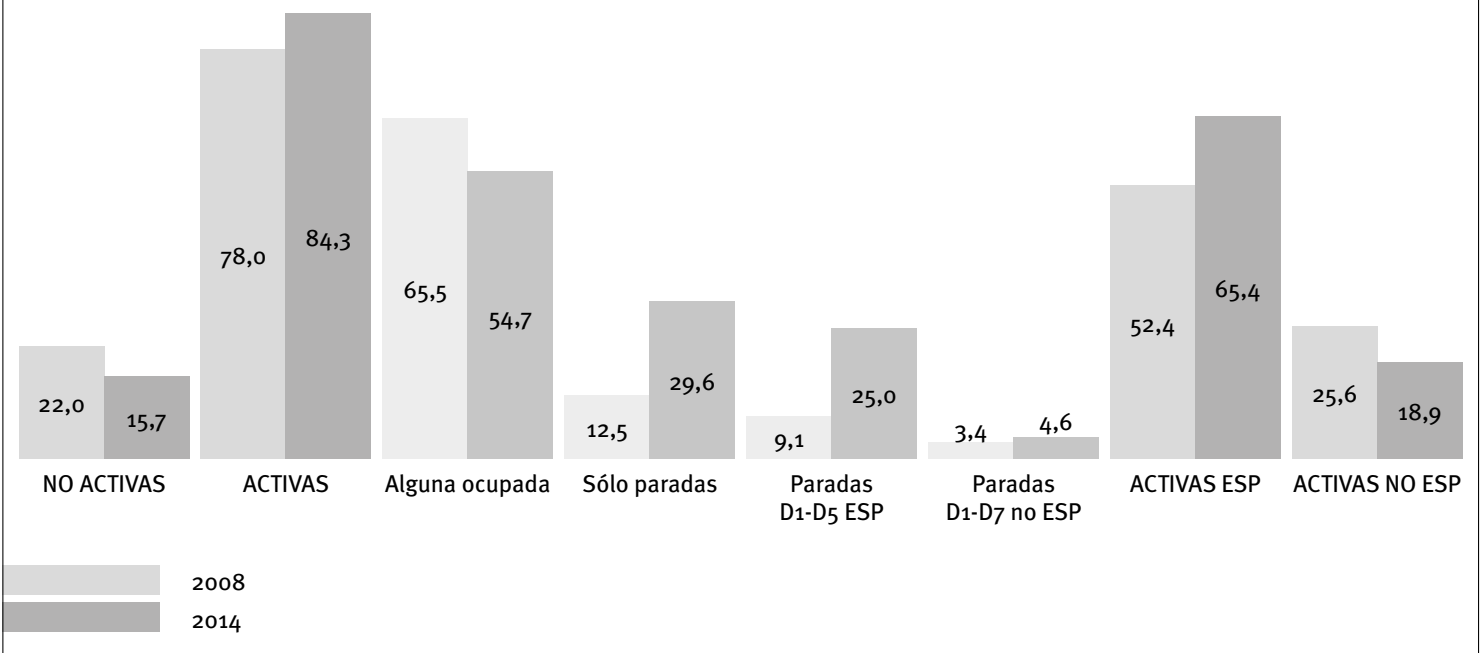

D: decil. ESP: nacidas en España. No ESP: no nacidas en España.

Fuente: Elaboración propia a partir de la Encuesta de Condiciones de Vida 2009 y 2015 (Instituto Nacional de Estadística, datos de renta de 2008 y 2014). 
alguna persona ocupada. Las personas que residen en estos hogares componen un $51,1 \%$ de la población en el decil 1 y un $54,7 \%$ de los que componen los deciles 1 a 3. Sin embargo, su peso se reduce en 2014 respecto al $58,4 \%$ y el $65,5 \%$ de 2008 , respectivamente.

- Aumenta, en cambio, de forma llamativa la proporción de personas en hogares con todos sus miembros desempleados. Esto es evidente en el decil 1, donde estas personas pasan de representar un $23 \%$ en 2008 a un 42,8\% en 2014 . En los deciles 1 a 3, el aumento es del 12,5\% al $29,6 \%$.

- Finalmente, en términos de lugar de nacimiento llama la atención el papel creciente, entre los grupos desfavorecidos, de las personas en hogares con población activa nacida en España. Este grupo ve aumentar su peso en el decil 1 del $47,7 \%$ al 60,1\% entre 2008 y 2014 . El aumento es del $52,4 \%$ al $65,4 \%$ en los deciles 1 a 3.

\section{El papel de los ingresos de las personas activas en ciertas comunidades autónomas}

Una de las características del actual modelo socioeconómico español es el notable esfuerzo realizado en la mejora de la posición de la población inactiva, una política que se enfrentará pronto a fuertes límites económicos, sociales y políticos, dado el contexto de rápido aumento de la población con acceso al sistema de pensiones. El peso que suponen los ingresos del colectivo en relación con los grupos de población activa más joven (menores de 45 años) muestra la fuerte dependencia económica que la población inactiva representa en la economía de algunas comunidades autónomas (Gráfico 10).

Gráfico 10. Relación entre los ingresos totales de los hogares de personas inactivas y los de los hogares de personas activas menores de 45 años. España, 2014 (\%)

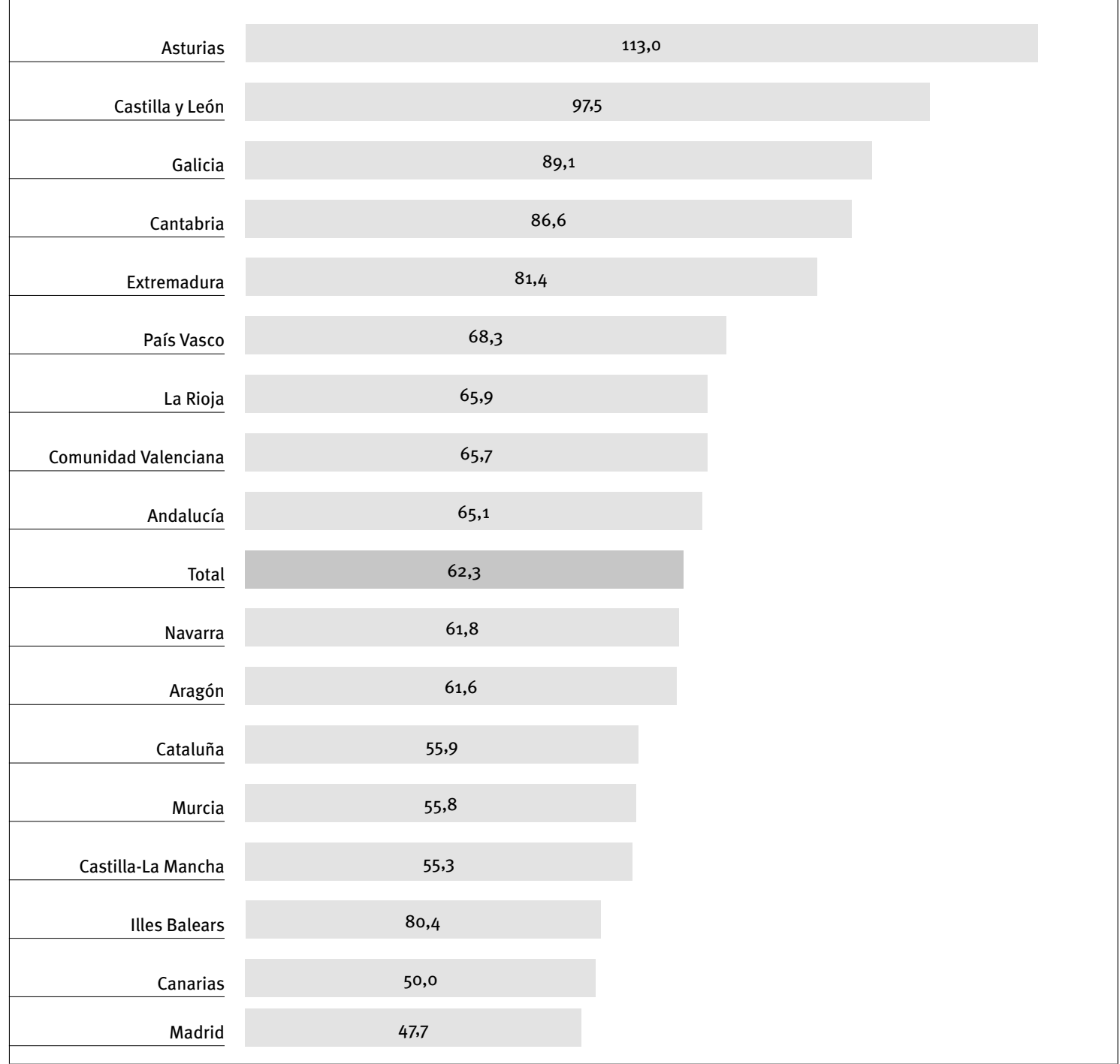

Fuente: Elaboración propia a partir de la Encuesta de Condiciones de Vida 2015 (Instituto Nacional de Estadística, datos de renta de 2014). 
Esta realidad es evidente en aquellas comunidades autónomas en las que el peso económico de los hogares de población inactiva es igual o superior al $80 \%$ de los ingresos de los hogares donde todos sus miembros son activos y menores de 45 años. En ese grupo, se incluye la parte noroccidental envejecida de España, incluyendo a las comunidades autónomas de Galicia, Asturias, Cantabria, y Castilla y León, además de Extremadura. En estas zonas, el bienestar regional es extremadamente dependiente de las pensiones.

\section{Principales resultados}

Los principales resultados del estudio son los siguientes:

- La crisis se caracteriza por una brutal y generalizada caída de los ingresos de los hogares con población activa, en completo contraste con la tendencia favorable de los hogares de población inactiva.

- La caída de ingresos alcanza niveles superiores al $30 \%$ en los grupos más desfavorecidos (decil 1 en los hogares de población nacida en España, y deciles 1 y 2 en aquellos donde está presente población activa nacida fuera de España).

- Aunque la población de origen extranjero sufre más intensamente la crisis, el grueso del proceso afecta a hogares de población nacida en España (supone el $81,1 \%$ de la caída total acumulada de ingresos).

- La caída de ingresos en los hogares de personas activas se traduce en un sustancial deterioro de las condiciones de vida, aunque de distinto carácter:

- En la parte más favorecida de la población española, apenas se observa deterioro en los indicadores ligados a las necesidades básicas. Sin embargo, aumentan en alrededor de 7 u 8 puntos los indicadores de inseguridad económica y de carencia en aspectos ligados al bienestar (posibilidad de disfrutar de vacaciones, por ejemplo).

- En los grupos menos favorecidos, tanto el intermedio como el peor situado en la escala de ingresos, el nivel de los indicadores asociados a la cobertura de las necesidades básicas se multiplica prácticamente por dos durante la crisis. Los problemas de inseguridad económica aumentan en más de 20 puntos. En el grupo intermedio, la población afectada por esta forma de inseguridad pasa de ser minoritaria en 2008 a claramente mayoritaria en 2014.

A pesar de la mejoría económica, el impacto social de la crisis sigue siendo evidente en 2015. Los problemas graves en la cobertura de las necesidades básicas siguen afectando a un porcentaje muy alto de personas de los siguientes grupos:

- Población en hogares de personas activas todas ellas desempleadas, pertenecientes a hogares con personas nacidas en el extranjero 0 a los deciles 1 a 5 de la población nacida en España: $40,6 \%$ de personas afectadas.

- En caso de presencia de personas ocupadas y paradas, el grupo equivalente, reducido a los deciles 1 y 2 en el caso de población residente en hogares nacionales, tiene un porcentaje de población con carencias del $27,1 \%$.

- En el decil 1 de población en hogares de personas inactivas, la proporción de personas con carencia es aún elevado: $18,5 \%$.

- Lo mismo ocurre en otros hogares de población activa nacida en España, en concreto en los deciles 3 a 6 de hogares de población con personas tanto ocupadas como paradas, así como en los deciles 6 a 8 de personas activas todas ellas desempleadas: un $14,5 \%$ de personas con problemas.

Finalmente, la evolución de los ingresos ha provocado un significativo cambio en la estructura interna de los grupos más desfavorecidos, los que componen los deciles 1 a 3. Los colectivos de población activa suponen, en la actualidad, más del $80 \%$ de la población en esos deciles, con un peso creciente en el decil 1 de la población en hogares con todas las personas desempleadas (un 42,8\% del total, frente a apenas un $23 \%$ en 2008). 
Anexo. Evolución de la renta, por grupos sociales según actividad, posición en la distribución de los ingresos y lugar de nacimiento. Población en cada grupo, renta media y renta acumulada total. España, 2008-2014

\begin{tabular}{|c|c|c|c|c|c|c|c|c|c|}
\hline \multirow[b]{2}{*}{ Grupo } & \multicolumn{3}{|c|}{2008} & \multicolumn{3}{|c|}{2014} & \multicolumn{3}{|c|}{$\Delta$ 2008-2014 (\%) } \\
\hline & $\begin{array}{l}\text { Población } \\
\text { (habs.) }\end{array}$ & $\begin{array}{c}\text { Renta } \\
\text { media (€) }\end{array}$ & Renta total (€) & $\begin{array}{l}\text { Población } \\
\text { (habs.) }\end{array}$ & $\begin{array}{c}\text { Renta } \\
\text { media }(€)\end{array}$ & Renta total & $\begin{array}{c}\text { Población } \\
\text { (habs.) }\end{array}$ & $\begin{array}{c}\text { Renta } \\
\text { media } \\
(€)\end{array}$ & $\begin{array}{c}\text { Renta } \\
\text { total } \\
(€)\end{array}$ \\
\hline No activas D1 & 691.820 & $3.978,63$ & $2.752 .499 .251,00$ & 710.760 & $4 \cdot 377,81$ & $3.111 .568 .842,83$ & 2,7 & 10,0 & 13,0 \\
\hline No activas D2-D5 & 2.763 .618 & $8.947,76$ & $24 \cdot 728.197 \cdot 371,67$ & 2.847 .859 & $10.050,96$ & $28.623 .717 .500,65$ & 3,0 & 12,3 & 15,8 \\
\hline No activas D6-D10 & 3.454 .497 & $20.005,44$ & $69.108 \cdot 745 \cdot 357,34$ & 3.559 .906 & $21.201,77$ & $75 \cdot 476.303 \cdot 441,78$ & 3,1 & 6,0 & 9,2 \\
\hline No activas (total) & 6.909 .936 & $13.978,34$ & $96.589 .441 .980,01$ & 7.118 .525 & $15.060,93$ & $107.211 .589 .785,25$ & 3,0 & 7,7 & 11,0 \\
\hline Activas ESP D5-D10 & $19.045 \cdot 585$ & $24.746,21$ & $471.306 .066 .728,14$ & 19.261 .648 & $22.196,27$ & $427 \cdot 536.753 \cdot 314,72$ & 1,1 & $-10,3$ & $-9,3$ \\
\hline $\begin{array}{l}\text { Activas no ESP } \\
\text { D8-D10 }\end{array}$ & 2.163 .237 & $22.710,47$ & $49.128 .140 .511,29$ & 2.028 .812 & $21.442,02$ & $43.501 .824 .903,93$ & $-6,2$ & $-5,6$ & $-11,5$ \\
\hline $\begin{array}{l}\text { Activas ESP D5-D10 } \\
\text { Activas no ESP } \\
\text { D8-D10 }\end{array}$ & 21.208 .822 & $24.538,57$ & $520.434 \cdot 207.239,42$ & 21.290 .460 & $22.124,40$ & $471.038 .578 .218,64$ & 0,4 & $-9,8$ & $-9,5$ \\
\hline Activas ESP D2 & 3.171 .605 & $8.994,72$ & $28.527 .694 .587,09$ & 3.218 .111 & $6.892,21$ & $22.179 .890 .844,10$ & 1,5 & $-23,4$ & $-22,3$ \\
\hline Activas ESP D3-D4 & 6.347 .217 & $12.398,80$ & $78.697 .869 .066,28$ & 6.416 .337 & $10.258,52$ & $65.822 .147 .026,37$ & 1,1 & $-17,3$ & $-16,4$ \\
\hline Activas no ESP D3 & 685.266 & $7.073,76$ & $4.847 .404 .734,50$ & 691.151 & $5.403,56$ & $3.734 .677 .763,57$ & 0,9 & $-23,6$ & $-23,0$ \\
\hline $\begin{array}{l}\text { Activas no ESP } \\
\text { D4-D7 }\end{array}$ & 2.893 .494 & $11.145,10$ & $32.248 .272 .614,40$ & 2.682 .087 & $9.401,23$ & $25.214 .927 .079,57$ & $-7,3$ & $-15,6$ & $-21,8$ \\
\hline $\begin{array}{l}\text { Activas ESP D2-D4 } \\
\text { Activas no ESP } \\
\text { D3-D7 }\end{array}$ & 13.097 .583 & $11.018,92$ & $144.321 .241 .002,27$ & 13.007 .687 & $8.990,96$ & $116.951 .642 .713,61$ & $-0,7$ & $-18,4$ & $-19,0$ \\
\hline Activas ESP D1 & 3.178 .791 & $4.751,20$ & $15.103 .084 .485,57$ & 3.213 .159 & $3.165,80$ & $10.172 .219 .850,62$ & 1,1 & $-33,4$ & $-32,6$ \\
\hline $\begin{array}{l}\text { Activas no ESP } \\
\text { D1-D2 }^{-D_{2}}\end{array}$ & 1.475 .165 & $3.589,82$ & $5.295 \cdot 573.692,95$ & 1.356 .549 & $2.352,48$ & $3.191 .255 .409,27$ & $-8,0$ & $-34,5$ & $-39,7$ \\
\hline $\begin{array}{l}\text { Activas ESP D1 } \\
\text { Activas no ESP } \\
\text { D1-D2 }\end{array}$ & 4.653 .956 & $4.383,08$ & $20.398 .658 .178,52$ & 4.569 .709 & $2.924,36$ & $13.363 .475 .259,90$ & $-1,8$ & $-33,3$ & $-34,5$ \\
\hline Activas ESP & 31.743 .198 & $18.701,16$ & $593.634 .714 .867,08$ & 32.109 .255 & $16.372,57$ & $525.711 .011 .035,81$ & 1,2 & $-12,5$ & $-11,4$ \\
\hline Activas no ESP & 7.217 .162 & $12.680,80$ & $91.519 \cdot 391.553,14$ & 6.758 .600 & $11.192,06$ & $75.642 .685 .156,34$ & $-6,4$ & $-11,7$ & $-17,3$ \\
\hline Activas (total) & 38.960 .360 & $17.585,93$ & $685 \cdot 154 \cdot 106.420,22$ & 38.867 .855 & $15 \cdot 471,75$ & $601.353 .696 .192,14$ & $-0,2$ & $-12,0$ & $-12,2$ \\
\hline Total & 45.870 .296 & $17.042,48$ & $781.743 \cdot 548.400,24$ & 45.986 .380 & $15 \cdot 408,16$ & $708.565 .285 \cdot 977,39$ & 0,3 & $-9,6$ & $-9,4$ \\
\hline
\end{tabular}

D: decil. ESP: nacidas en España. No ESP: no nacidas en España.

Fuente: Elaboración propia a partir de la Encuesta de Condiciones de Vida 2009 y 2015 (Instituto Nacional de Estadística, datos de renta de 2008 y 2014). 


\title{
Bibliografía referenciada
} crecido?", El País, 22-11-16 [khttp:// elpais.com/elpais/2016/11/02/
opinion/1478079292_689770.html>]

INSTITUTO NACIONAL DE ESTADÍSTICA: Encuesta de

\author{
Condiciones de Vida.
}

